\title{
Isolation of Endophytic Fungi from Tridax Procumbens Grown in Contaminated Sites and Assessment of Their Heavy Metal Tolerance
}

\author{
Arul Selvi. C. ${ }^{1}$, Soundhari C. ${ }^{2 *}$ \\ ${ }^{1}$ Post Graduate student, ${ }^{2}$ Associate Professor, Department of Microbiology, Valliammal College \\ for Women, E9, Anna Nagar East, Chennai - 102, Tamil Nadu, India
}

\begin{abstract}
Seven strains of endophytic fungi were isolated from the leaves of Tridax procumbens and were first screened individually for tolerance to heavy metals like $\mathrm{Zn}, \mathrm{Pb}, \mathrm{Cu}, \mathrm{Mg}$, and $\mathrm{Cr}$ from $5 \mathrm{ppm}$ to $50 \mathrm{ppm}$ on Potato dextrose agar medium. They were further tested for resistance at $75,100,200,400$, and 600 ppm of heavy metals. The fungal isolate on the PDA medium without any heavy metal was used as a control for growth comparison. It was observed that with increasing concentration of heavy metal from 5 to $600 \mathrm{ppm}$ there was a decrease in the number of fungal isolates. Few of the fungal isolates were able to tolerate heavy metals up to $600 \mathrm{ppm}$. The screening test revealed heterogeneity in the heavy metal tolerance of the isolates.
\end{abstract}

Keywords: Heavy metal tolerance, endophytic fungi, Tridax procumbens, metal pollution

\section{Introduction}

Heavy metals are high density metals that are toxic even at ppb levels. Heavy metal pollution is a major problem in the environment all over the world. Heavy metals are continuously discharged into the environment due to various industrial activities. Heavy metals like $\mathrm{Cd}, \mathrm{Cr}, \mathrm{Co}, \mathrm{Cu}, \mathrm{Pb}$, $\mathrm{Ni}, \mathrm{Hg}$, and $\mathrm{Zn}$ are considered as the most hazardous metals by the World Health Organization (WHO) [1]. A soil with the natural composition of heavy metal does not affect animals or plants. Heavy metals like $\mathrm{Zn}, \mathrm{Pb}, \mathrm{Cu}, \mathrm{Mg}$, and $\mathrm{Cr}$ when released above certain threshold levels can contaminate and be toxic to the soil, surface water, groundwater and sediments [2] and can exert certain deleterious effects on the biological system. They cannot undergo biodegradation and are hence in living organisms, leading to various diseases and disorders even at low concentrations.

*Author for Correspondence. E-mail: drcsoundhari@gmail.com

This work is licensed under a Creative Commons Attribution-Noncommercial-Share Alike 4.0 International License. 
These metals cause damage to brain, can lead to other effects like reproductive failures, nervous system failures, and can also induce tumour formation in human beings [3].

Heavy metal pollution can be studied by the estimation of the total number of tolerant microorganisms. The bacteria and fungi isolated from polluted environments showed high-level tolerance to metals when compared to bacteria and fungi isolated from unpolluted environments [4]. Bioremediation of heavy metals can be best attained by the use of endophytic organisms. Fungal endophytes live in a mutualistic relationship with plant host for at least a part of their life, without causing any disease. The resistance of endophytic fungi to heavy metals and their efficiency for the removal of heavy metals differs greatly. T. procumbens is a widespread weed seen throughout the year in India [5] which removes effectively heavy metals like $\mathrm{Cr}, \mathrm{Cu}, \mathrm{Ni}, \mathrm{Pb}$, and Cd from soil contamination [6]. T. procumbens belongs to Asteraceae family and is a is a perennial plant. This species found usage in ayurvedic medicine in ancient times. [7]. It is common in tropical or semitropical climate fields, croplands, lawns, and roadsides [8]. Several approaches are used for the reduction of heavy metal pollution which are found ineffective due to incomplete removal of heavy metals. Hence the investigation was aimed to isolate endophytic fungi from $T$. procumbens and to screen their metal tolerance and resistance level to few heavy metals under lab conditions.

\section{Materials and methods}

\section{Collection of sample:}

Five different Tridax procumbens samples were collected from different industrial polluted sites in Chennai city (Fig.1(a), 1(b)). The plants were carefully uprooted and collected using polythene bags. The confirmation of the plant was done at the Department of Plant Biology and Biotechnology Quaid-e-millath Government College, Chennai. The leaves of T. procumbens were separated were washed in running tap water.
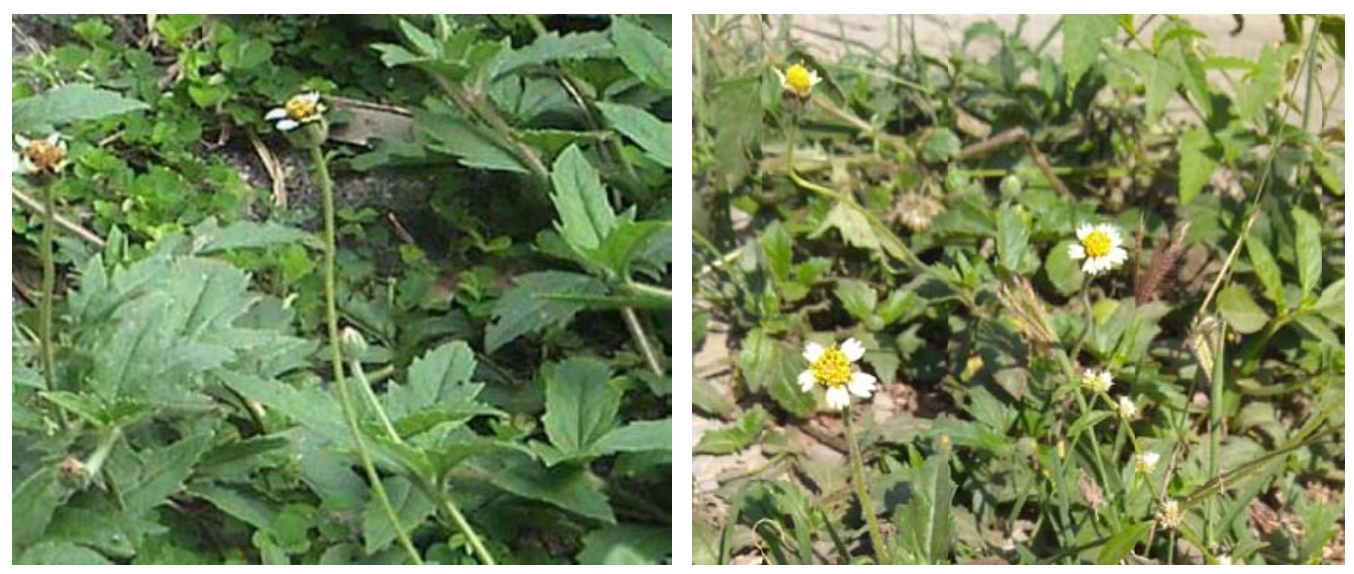

Fig. 1(a) and Fig.1(b): T. procumbens were collected from different industrial polluted sites. 


\section{Surface sterilization:}

The leaves were washed using sterile distilled water for 2 to 3 times and rinsed thoroughly with $75 \%$ ethanol (Fig.2a). After surface sterilization, the leaves were air-dried in sterile conditions (Fig.2b).

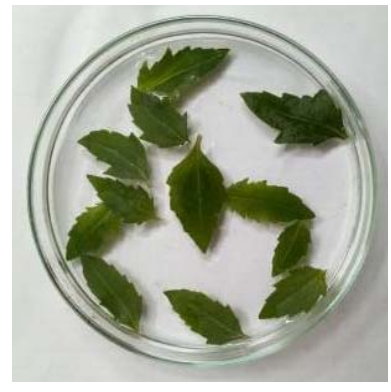

Fig.2(a)

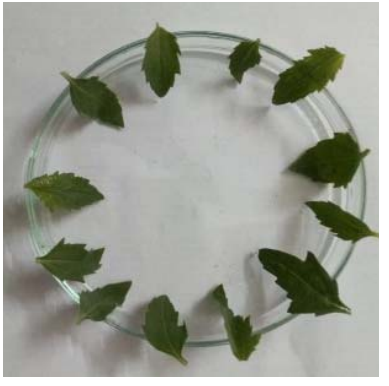

Fig.2(b)

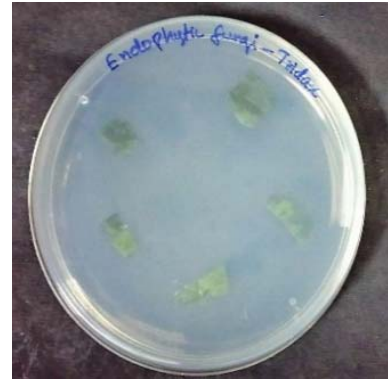

Fig.2(c)

Fig.2 (a, b \& c): Surface sterilized T. procumbens and leaves plated in PDA

\section{Isolation and Identification of Endophytic Fungi:}

Potato Dextrose Agar (PDA) medium supplemented with chloramphenicol was used as a culture medium. The freshly prepared medium was sterilized and under aseptic conditions was poured to sterile petriplates and allowed to cool. The surface sterilized leaves of $T$. procumbens were placed on to sterile PDA plates (Fig.2c) and, incubated at $25^{\circ} \mathrm{C}$ for 4 to 6 days. The mycelia of dominant isolates were transferred to a fresh PDA medium to obtain pure fungi strains. Pure isolates of endophytic fungi were characterized by the macroscopic appearance and microscopic appearance.

\section{Heavy metal resistant endophytic fungal selection:}

Isolated endophytic fungi were screened individually for heavy metal tolerance at concentrations from 5 to $50 \mathrm{ppm}$ to Zinc chloride $(\mathrm{Zn})$, Lead acetate $(\mathrm{Pb})$, Cupric sulphate $(\mathrm{Cu})$, Magnesium chloride (Mg), and Potassium dichromate (Cr). PDA medium inoculated with the isolate without any heavy metal is served as the control for comparison of fungal growth. Observations were recorded in comparison to control as presence or absence of growth. Heavy metal-tolerant (50ppm) fungal isolates were further tested for tolerance to increasing concentrations of 75, 100, 200, 400, and $600 \mathrm{ppm}$ of $\mathrm{Zn}, \mathrm{Pb}, \mathrm{Cu}, \mathrm{Mg}$, and $\mathrm{Cr}$ individually on PDA. The plates were inoculated with young fungal culture and it was incubated at $25^{\circ} \mathrm{C}$ for $7-14$ days [9], [10]. 


\section{Results}

\section{Isolation and identification of endophytic fungi:}

Seven dominant endophytic fungi were successfully isolated from the leaves of T. procumbens collected from different industrial polluted sites. Preliminary identification based on their morphological characteristics on PDA and microscopic examination of the isolates after staining with Lactophenol cotton blue (LPCB) revealed the isolates could be related to the following genera Fusarium sp. (strain 1 - Fusarium sp. (Fig.3.1), strain 2 - Fusarium sp. (Fig.3.2), Aspergillus sp. (strain 3 - A. flavus (Fig.3.3), strain 4 - A. niger (Fig.3.4), strain 5 - Curvularia (Fig.3.5), strain 6 and strain 7 (Fig.3.6 \& 3.7).

\section{Screening and determination of tolerance levels of endophytic fungal isolates to $\mathrm{Zn}$, $\mathrm{Pd}, \mathrm{Cu}, \mathrm{Mg}$, and $\mathrm{Cr}$ :}

The endophytic fungal isolates from T. procumbens collected from different industrial polluted sites were screened for tolerance from $5 \mathrm{ppm}$ to $600 \mathrm{ppm}$ concentration of each heavy metal (Zn, $\mathrm{Pb}, \mathrm{Cu}, \mathrm{Mg}$, and $\mathrm{Cr}$ ). All the isolates were tolerant of all the heavy metals at varying concentrations. Maximum tolerance was shown towards $\mathrm{Mg}$ by all isolated strains at $600 \mathrm{ppm}$ (table.1). Strain $1 \& 3$ showed high tolerance to $\mathrm{Zn}$ at $600 \mathrm{ppm}$ and strain 7 (400ppm), strain 2 (200ppm), strain 5 and 6 (100ppm). Strain 4 (50ppm) showed the lowest tolerance for $\mathrm{Zn}$. Maximum tolerant to $\mathrm{Cu}$ was showed by strain 3 at (600ppm), strain 1 and 5 (400ppm), strain 4 (200ppm), strain 2, 6 and 7 (100ppm). The highest tolerance to $\mathrm{Pb}$ was shown by strain 1, 3 and 4 at (600ppm), strain 5 and 7 (400ppm), and strain 2 and $6(200 \mathrm{ppm})$. Cr showed the strongest inhibition of growth. Strain 1, 3, 5 and 7, could tolerate a concentration of $50 \mathrm{ppm}$, and other strains to a lesser concentration strain 6 (25ppm), strain $2(10 \mathrm{ppm}) \&$ strain 4 (10ppm) shows minimum tolerance.

The ranges of heavy metal tolerance of all the fungal isolates are presented in Table 1. It was observed that with the increase in the concentration of heavy metal there was a decrease in the growth of the isolate. The heavy metal tolerance limits of fungal isolates are presented in (Fig. 4).

Fig. 3: Microscopic examination of isolated endophytic fungi

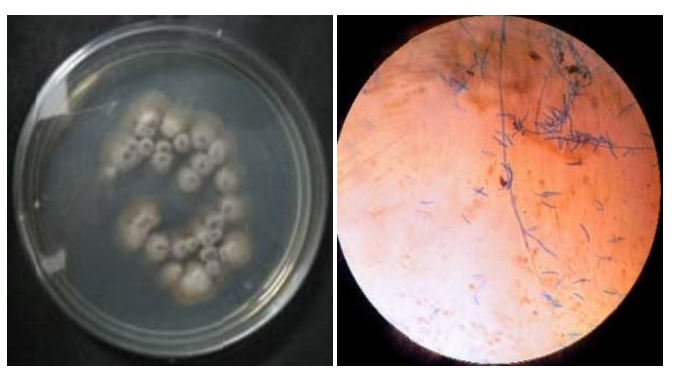

Fig.3.1 (a, b) Fusarium sp.

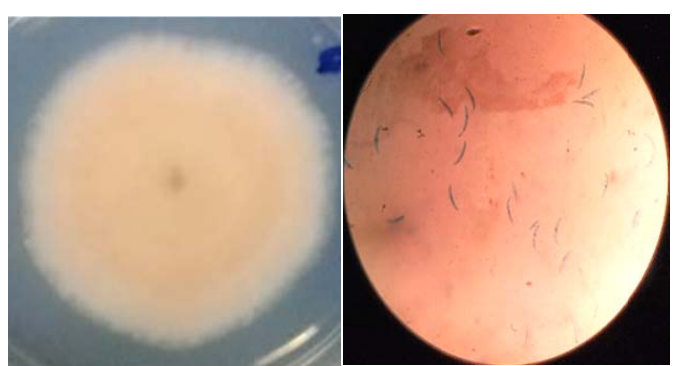

Fig.3.2 (a, b) Fusarium sp. 


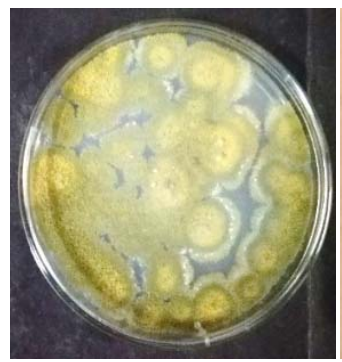

Fig.3.3 (a, b) A. flavus

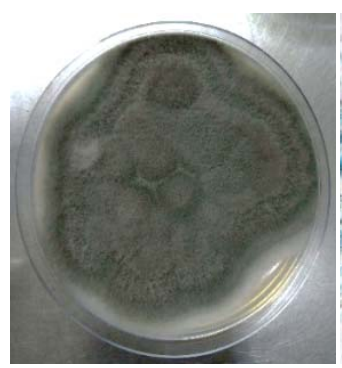

Fig.3.5 (a, b) Curvularia
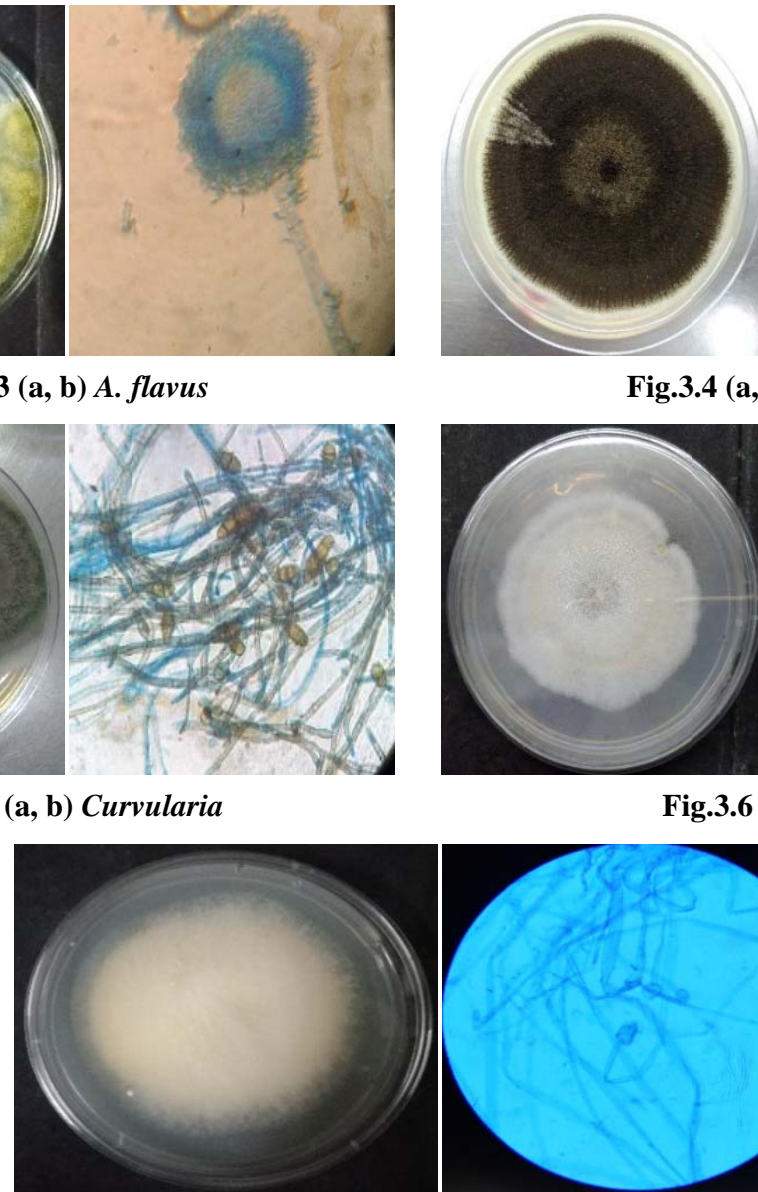

Fig.3.4 (a, b) A. niger
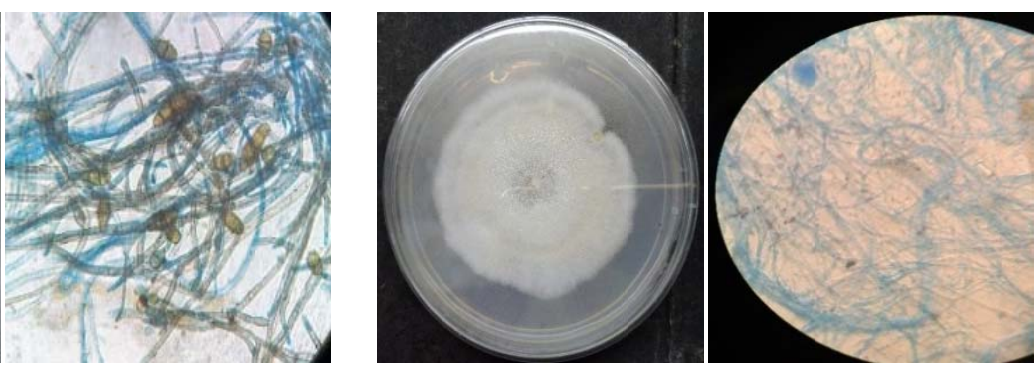

Fig.3.6 (a, b) S6

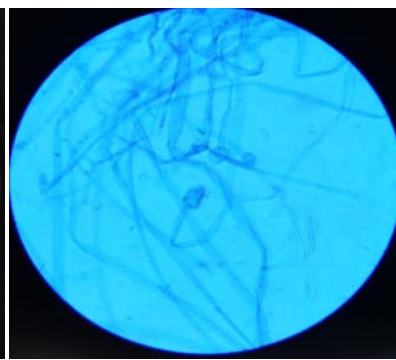

Fig.3.7 (a, b) S7

Table 1: Tolerance indices of Endophytic fungal isolates to heavy metals $\mathrm{Zn}, \mathrm{Pb}, \mathrm{Cu}, \mathrm{Mg}$, and $\mathrm{Cr}$.

\begin{tabular}{|c|c|l|l|l|l|}
\hline Strains & Zn & Pb & Cu & Mg & Cr \\
\hline S1 & $600 \mathrm{ppm}$ & $600 \mathrm{ppm}$ & $400 \mathrm{ppm}$ & $600 \mathrm{ppm}$ & $50 \mathrm{ppm}$ \\
\hline S2 & $200 \mathrm{ppm}$ & $200 \mathrm{ppm}$ & $100 \mathrm{ppm}$ & $600 \mathrm{ppm}$ & $10 \mathrm{ppm}$ \\
\hline S3 & $600 \mathrm{ppm}$ & $600 \mathrm{ppm}$ & $600 \mathrm{ppm}$ & $600 \mathrm{ppm}$ & $50 \mathrm{ppm}$ \\
\hline S4 & $50 \mathrm{ppm}$ & $600 \mathrm{ppm}$ & $200 \mathrm{ppm}$ & $600 \mathrm{ppm}$ & $10 \mathrm{ppm}$ \\
\hline S5 & $100 \mathrm{ppm}$ & $400 \mathrm{ppm}$ & $400 \mathrm{ppm}$ & $600 \mathrm{ppm}$ & $50 \mathrm{ppm}$ \\
\hline S6 & $100 \mathrm{ppm}$ & $200 \mathrm{ppm}$ & $100 \mathrm{ppm}$ & $600 \mathrm{ppm}$ & $25 \mathrm{ppm}$ \\
\hline S7 & $400 \mathrm{ppm}$ & $400 \mathrm{ppm}$ & $100 \mathrm{ppm}$ & $600 \mathrm{ppm}$ & $50 \mathrm{ppm}$ \\
\hline
\end{tabular}


GRAPH.1: Tolerance indices of isolated strains to various heavy metals $\mathrm{Zn}, \mathrm{Pb}, \mathrm{Cu}, \mathrm{Mg}$, and $\mathrm{Cr}$.

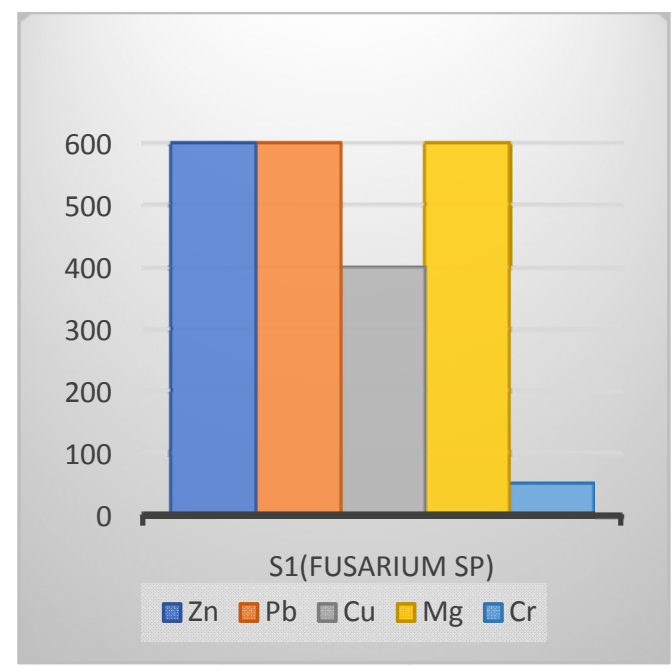

GRAPH.1(a)

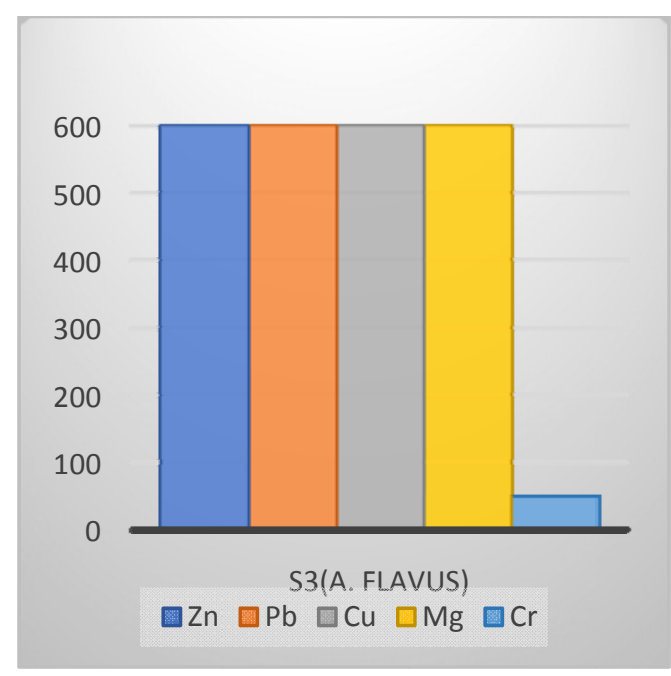

GRAPH.1(c)

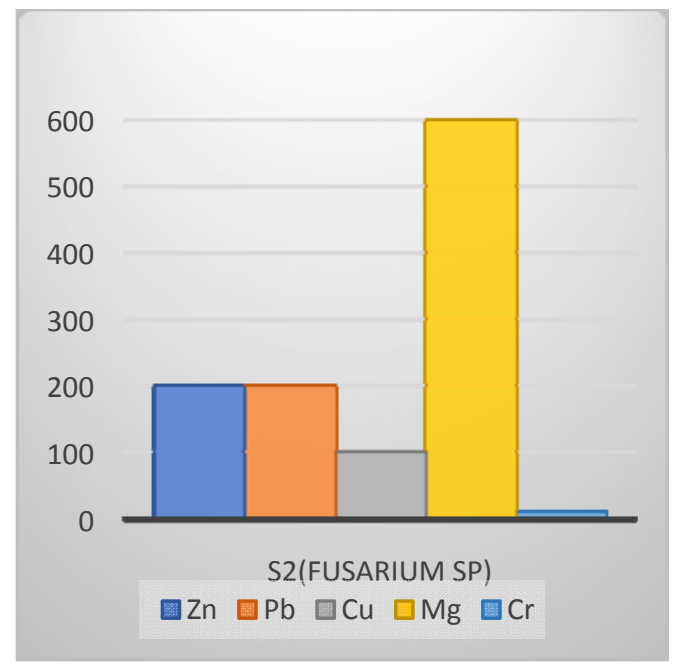

GRAPH.1(b)

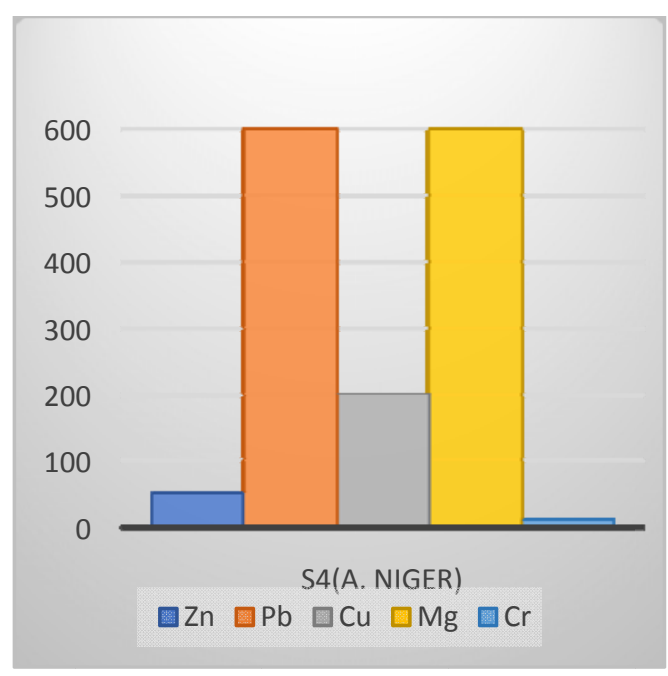

GRAPH.1(d) 
Isolation of Endophytic Fungi from Tridax Procumbens Grown in Contaminated Sites and ..... 7

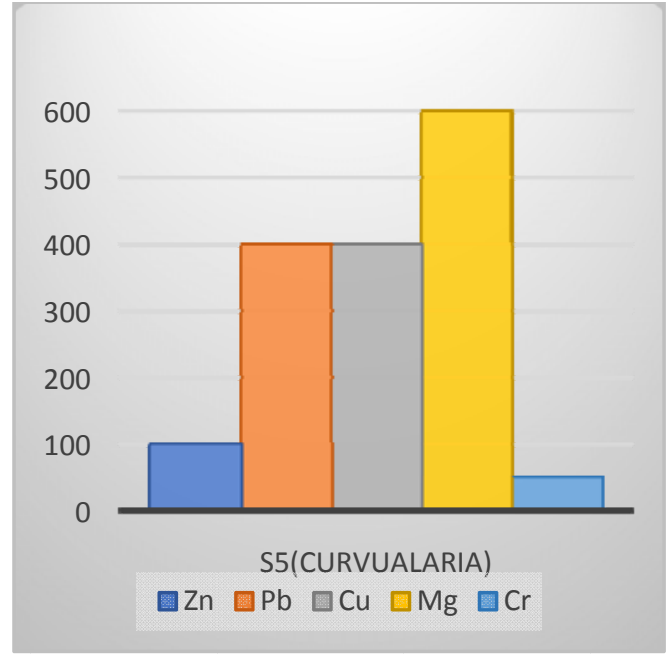

GRAPH.1(e)

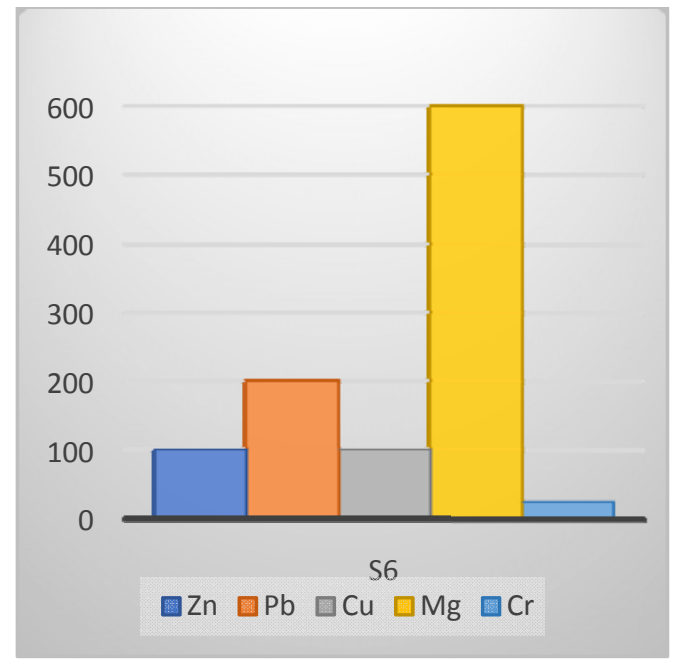

GRAPH.1(f)

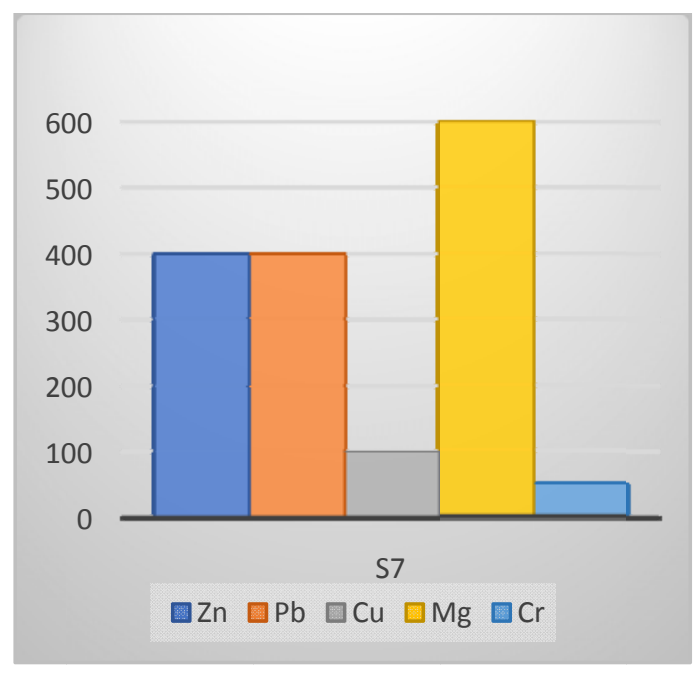

GRAPH.1(g) 
ARUL SELVI. C e tal

Fig.4.1: Growth of S1 (Fusarium sp.) after exposure to different concentrations of heavy metal ions for 7-14 days.

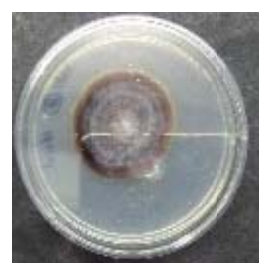

Control

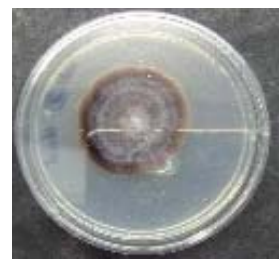

Control

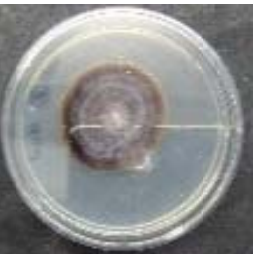

Control

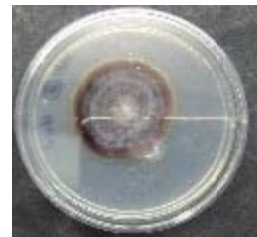

Control

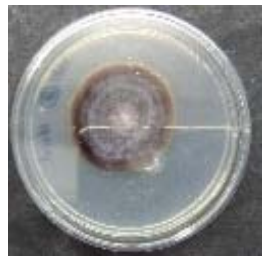

Control

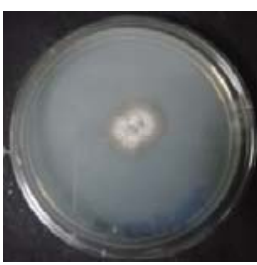

Zn(100ppm)

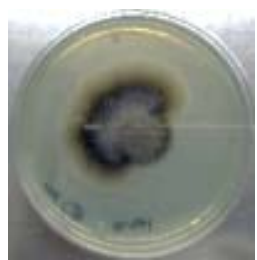

$\operatorname{Pb}(100 p p m)$

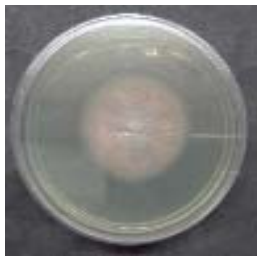

Mg(100ppm)

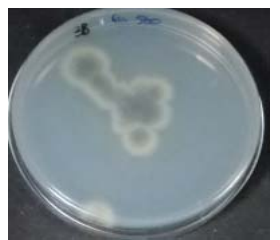

$\mathrm{Cu}(100 \mathrm{ppm})$

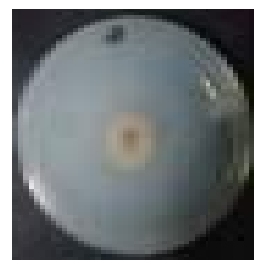

Zn(200ppm)

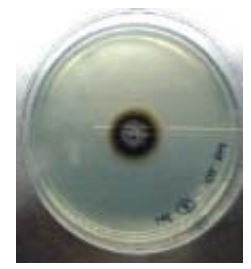

$\mathbf{P b}(200 \mathrm{ppm})$

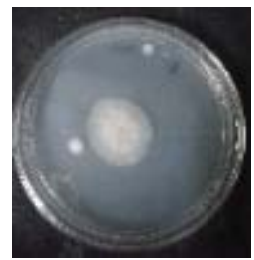

$\operatorname{Mg}(200 p p m)$

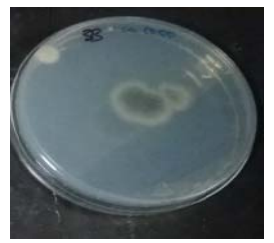

$\mathrm{Cu}(200 \mathrm{ppm})$

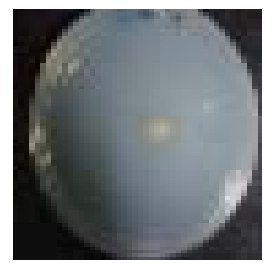

Zn(400ppm)

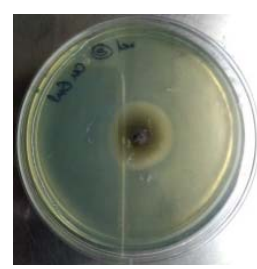

$\mathrm{Pb}(400 \mathrm{ppm})$

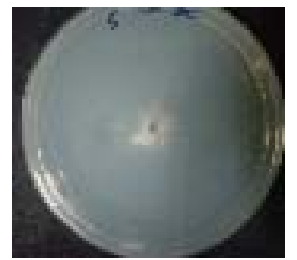

Mg(400ppm)

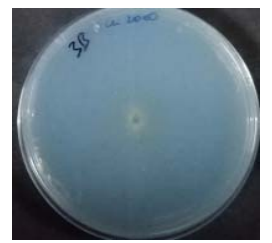

$\mathrm{Cu}(400 \mathrm{ppm})$

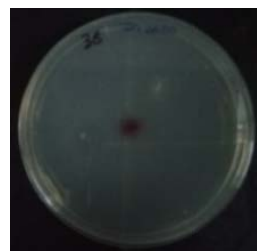

Zn(600ppm)

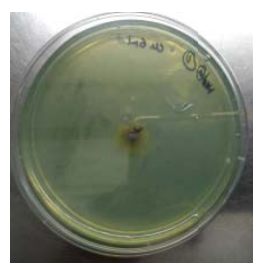

Pb(600ppm)

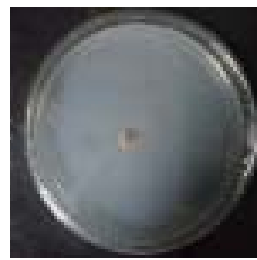

Mg(600ppm)

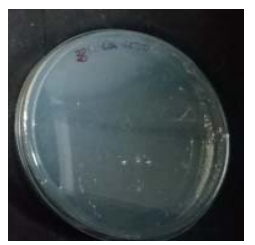

$\mathrm{Cu}(600 \mathrm{ppm})$

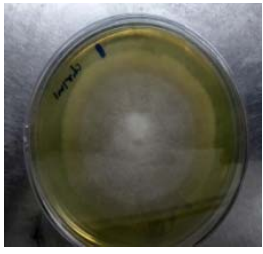

Cr(10ppm)

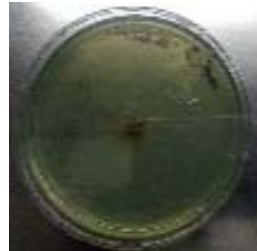

Cr(25ppm)

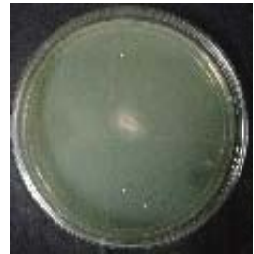

Cr(50ppm) 
Fig.4.2: Growth of S2 (Fusarium sp.) after exposure to different concentrations of heavy metal ions for 7-14 days.

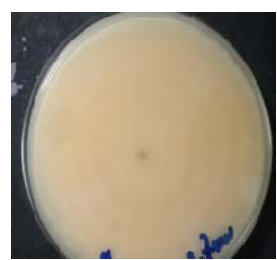

Control

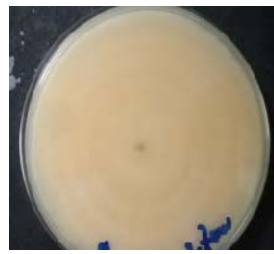

Control

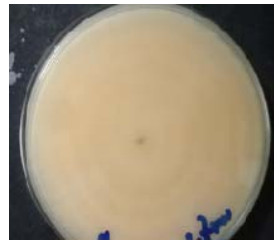

Control

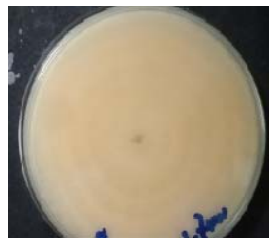

Control

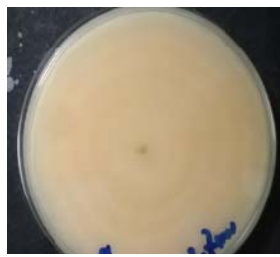

Control

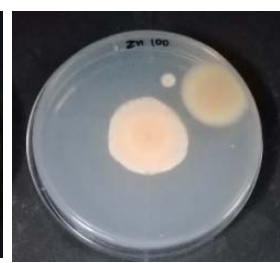

Zn(100ppm)

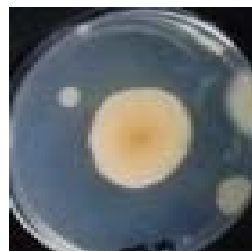

Pb(100ppm)

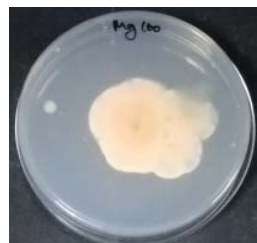

$\operatorname{Mg}(100 p p m)$

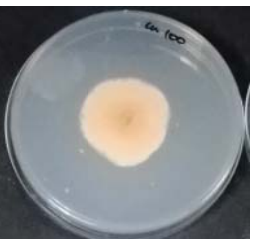

$\mathrm{Cu}(100 \mathrm{ppm})$

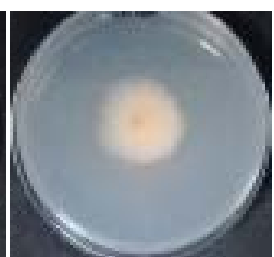

Zn(200ppm)

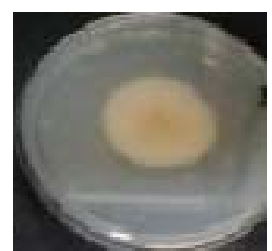

$\mathrm{Pb}(200 \mathrm{ppm})$

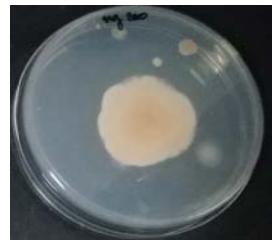

$\operatorname{Mg}(200 p p m)$

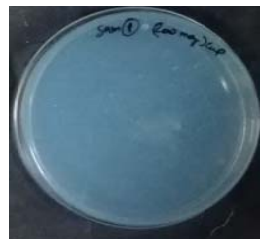

Cu(200ppm)

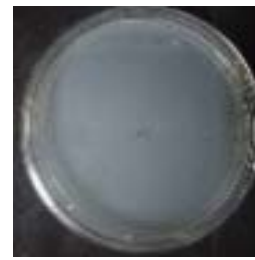

Zn(400ppm)

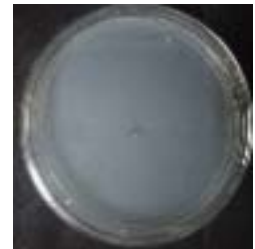

Pb(400ppm)

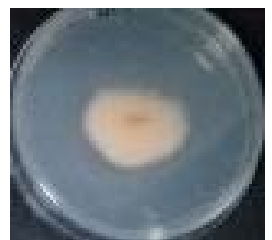

$\operatorname{Mg}(400 \mathrm{ppm})$

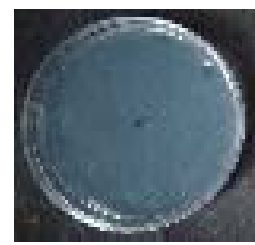

$\mathrm{Cu}(400 \mathrm{ppm})$

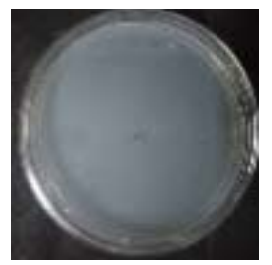

$\mathrm{Zn}(600 \mathrm{ppm})$

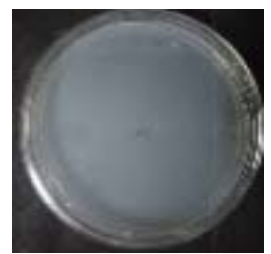

$\mathrm{Pb}(600 \mathrm{ppm})$

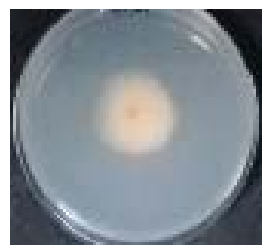

$\operatorname{Mg}(600 p p m)$

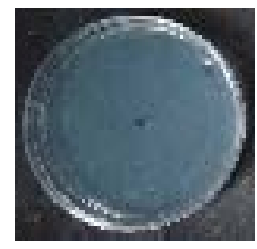

Cu(600ppm)

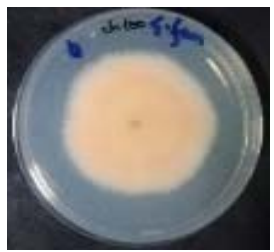

$\operatorname{Cr}(10 \mathrm{ppm})$

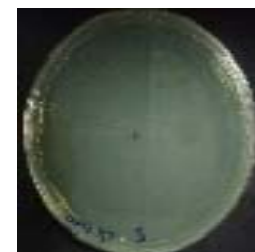

$\operatorname{Cr}(25 p p m)$

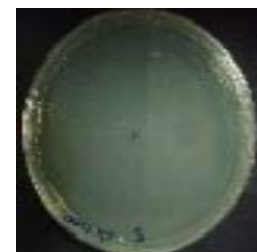

Cr(50ppm) 
Fig.4.3: Growth of S3 (A. flavus) after exposure to different concentrations of heavy metal ions for 7-14 days.

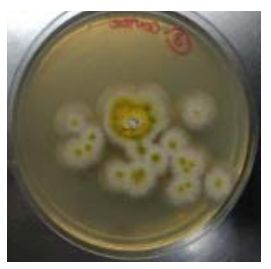

Control

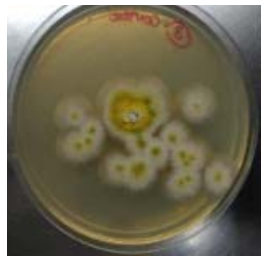

Control

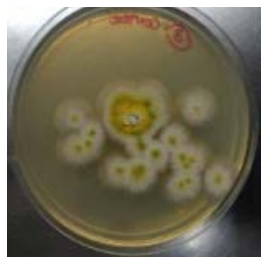

Control

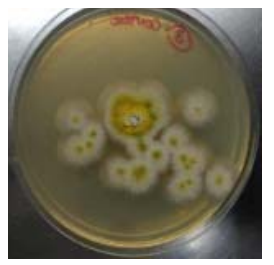

Control

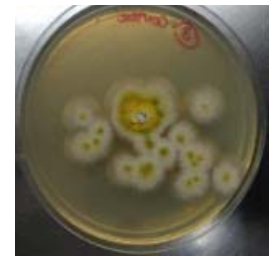

Control

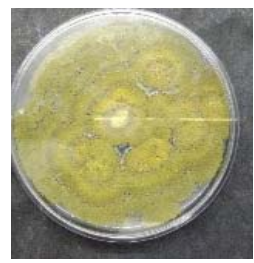

$\mathrm{Zn}(100 \mathrm{ppm})$

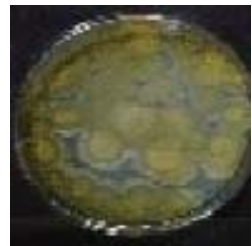

Pb(100ppm)

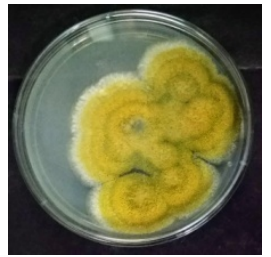

Cu(100ppm)

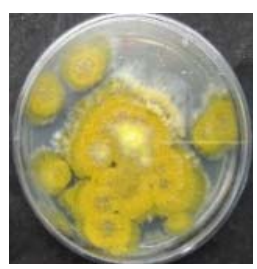

Mg(100ppm)

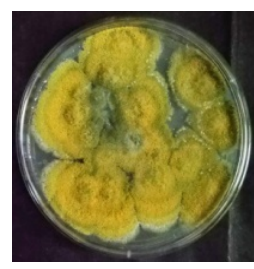

Zn(200ppm)

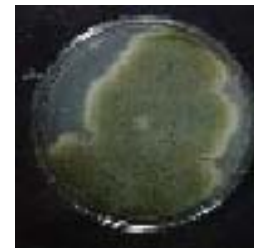

$\mathrm{Pb}(200 \mathrm{ppm})$

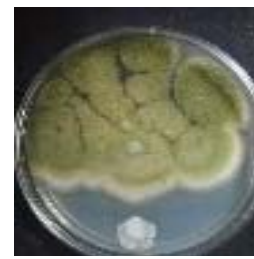

Cu(200ppm)

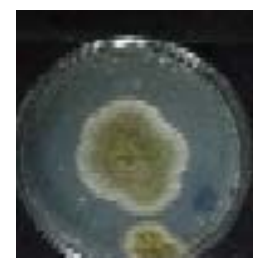

Mg(200ppm)

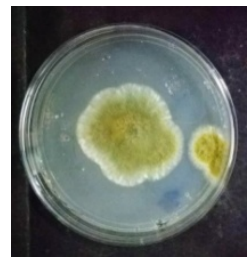

Zn(400ppm)

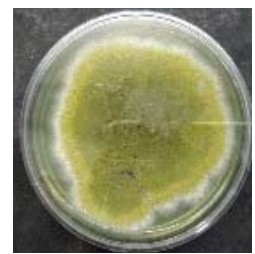

Pb(400ppm)

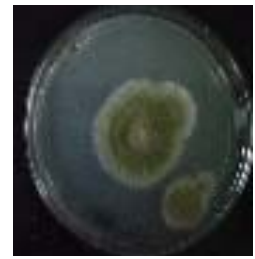

$\mathrm{Cu}(400 \mathrm{ppm})$

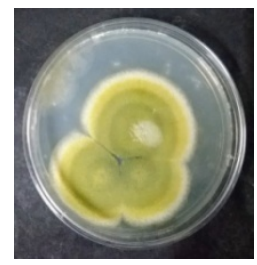

Mg(400ppm)

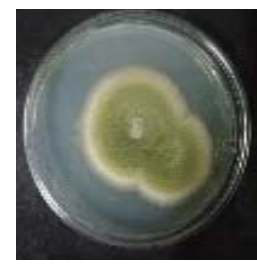

Zn(600ppm)

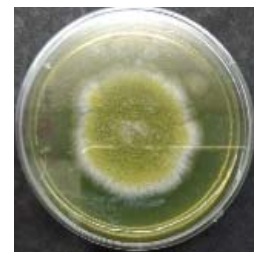

$\mathrm{Pb}$ (600ppm)

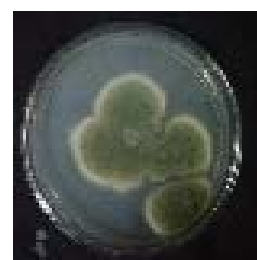

$\mathrm{Cu}(600 \mathrm{ppm})$

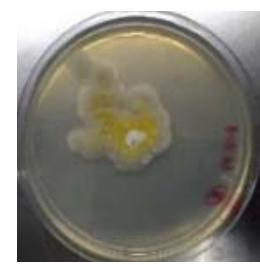

$\operatorname{Mg}(600 \mathrm{ppm})$

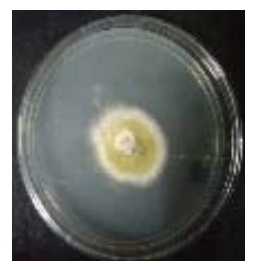

$\operatorname{Cr}(10 p p m)$

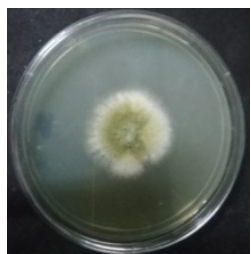

$\operatorname{Cr}(25 p p m)$

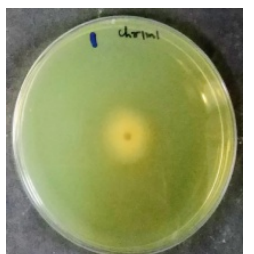

Cr(50ppm) 
Fig.4.4: Growth of S4 (A. niger ) after exposure to different concentrations of heavy metal ions for 7-14 days.

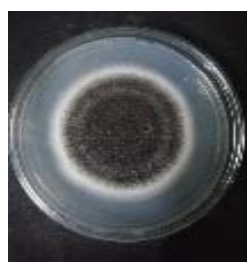

Control

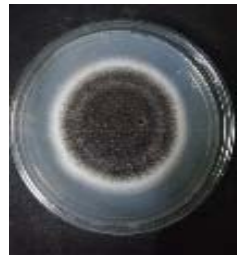

Control

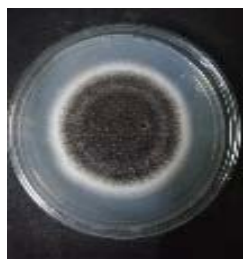

Control

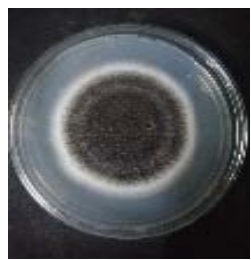

Control

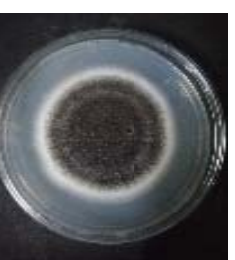

Control
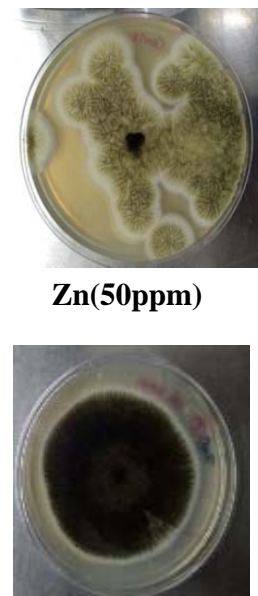

$\mathrm{Pb}(100 \mathrm{ppm})$

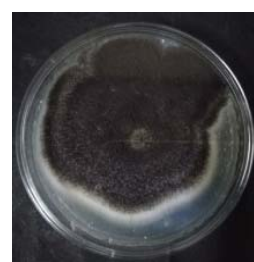

$\mathrm{Cu}(100 \mathrm{ppm})$

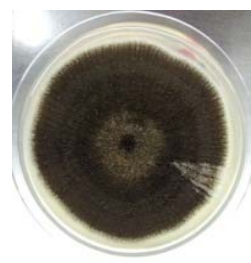

$\operatorname{Mg}(100 \mathrm{ppm})$

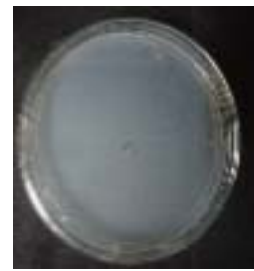

Zn(200ppm)

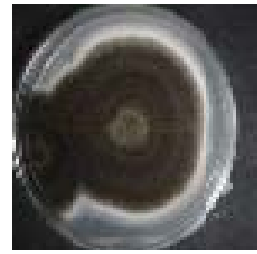

$\mathrm{Pb}(200 \mathrm{ppm})$

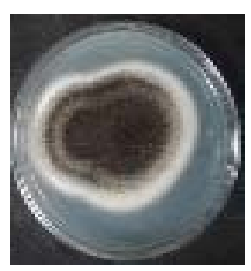

Cu(200ppm)

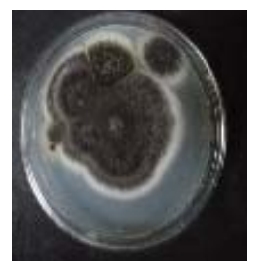

$\operatorname{Mg}(200 \mathrm{ppm})$

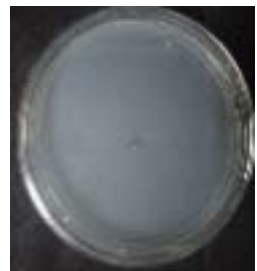

Zn(400ppm)

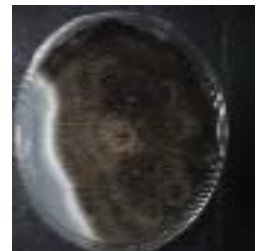

Pb(400ppm)

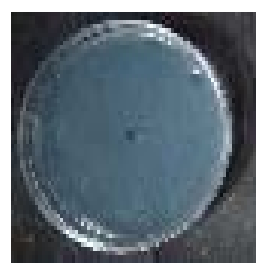

$\mathrm{Cu}(400 \mathrm{ppm})$

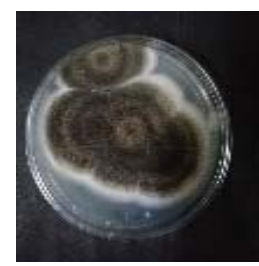

Mg(400ppm)

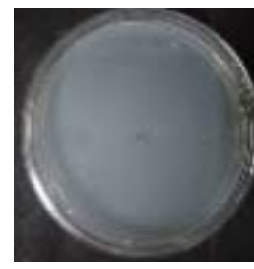

Zn(600ppm)

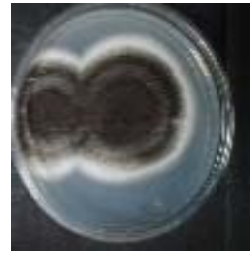

Pb(600ppm)

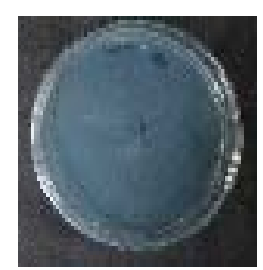

$\mathrm{Cu}(600 \mathrm{ppm})$

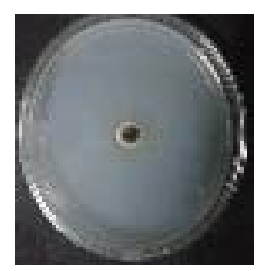

Mg(600ppm)

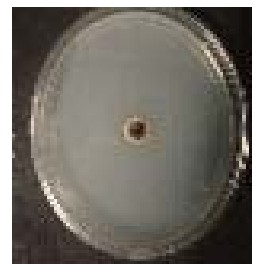

$\operatorname{Cr}(10 \mathrm{ppm})$

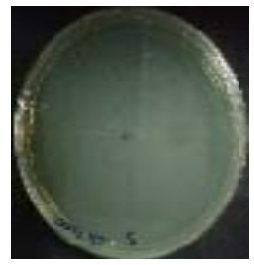

Cr(25ppm)

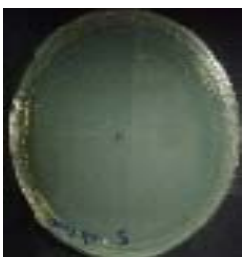

Cr(50ppm) 
Fig.4.5: Growth of S5 (Curvularia) after exposure to different concentrations of heavy metal ions for 7-14 days.

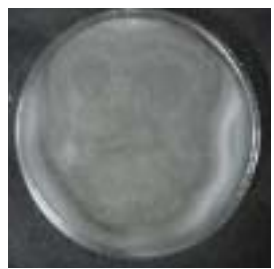

Control

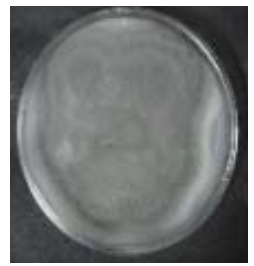

Control

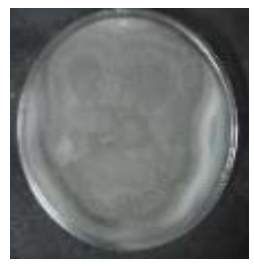

Control

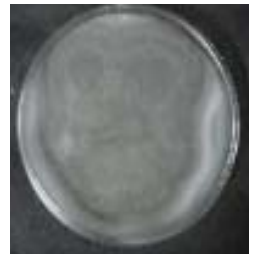

Control

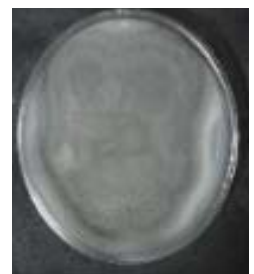

Control

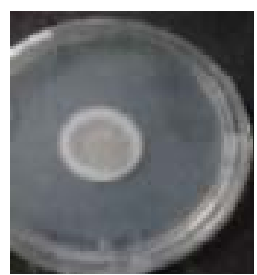

Zn(100ppm)

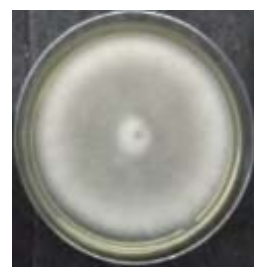

$\mathrm{Pb}(100 \mathrm{ppm})$

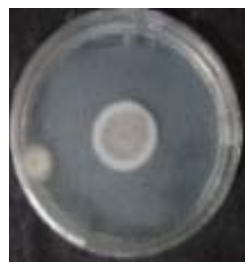

Cu(100ppm)

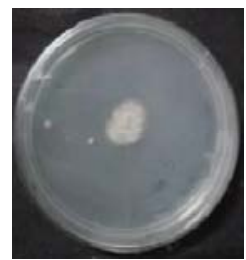

Mg(100ppm)

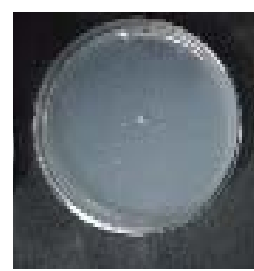

Zn(200ppm)

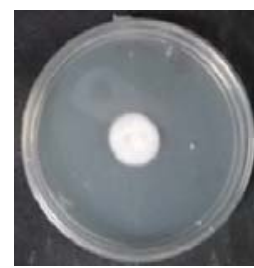

Pb(200ppm)

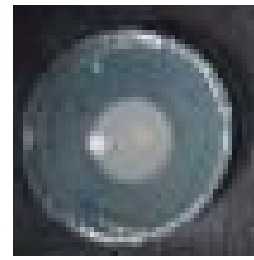

$\mathrm{Cu}(200 \mathrm{ppm})$

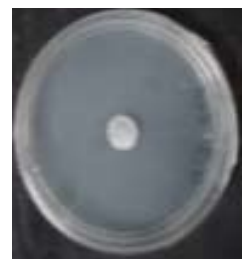

$\operatorname{Mg}(200 p p m)$

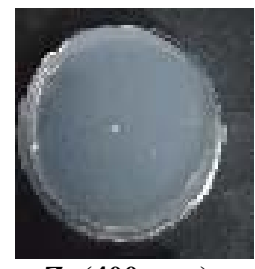

Zn(400ppm)

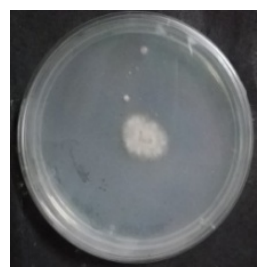

$\mathrm{Pb}(400 \mathrm{ppm})$

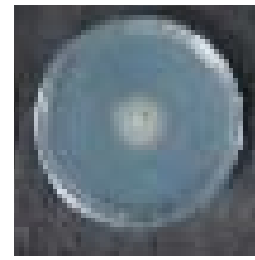

Cu(400ppm)

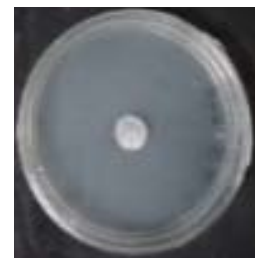

$\operatorname{Mg}(400 \mathrm{ppm})$
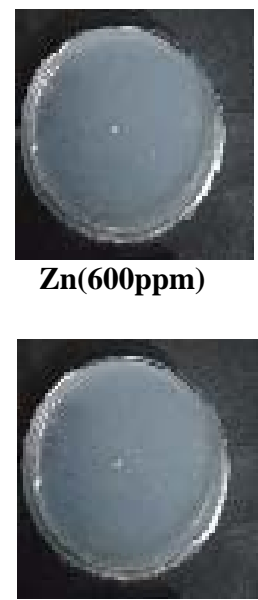

$\mathrm{Pb}(600 \mathrm{ppm})$

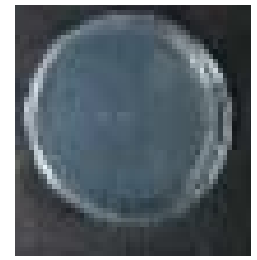

Cu(600ppm)

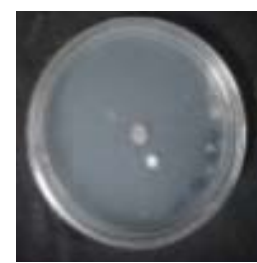

$\operatorname{Mg}(600 \mathrm{ppm})$

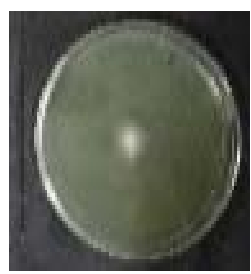

$\operatorname{Cr}(10 p p m)$

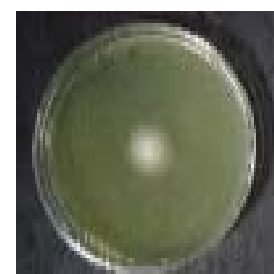

Cr(25ppm)

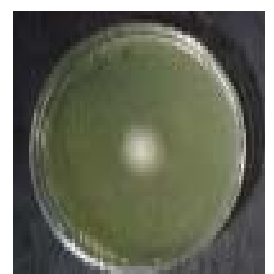

Cr(50ppm) 
Fig.4.6: Growth of S6 after exposure to different concentrations of heavy metal ions for 7-14 days.

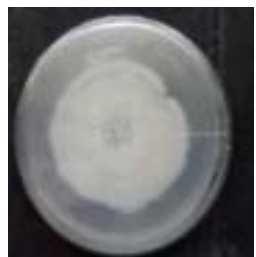

Control

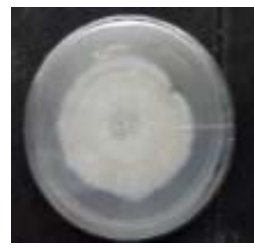

Control

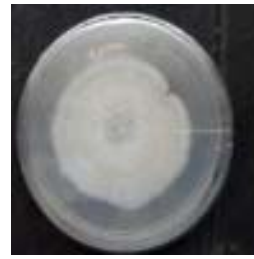

Control

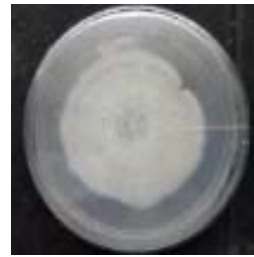

Control

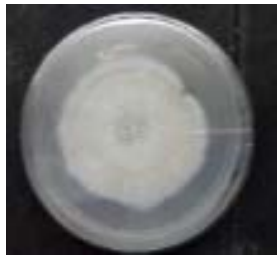

Control

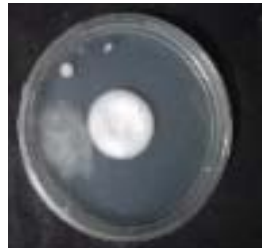

Zn(100ppm)

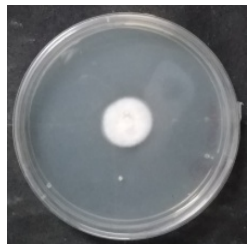

$\operatorname{Pb}(100 \mathrm{ppm})$

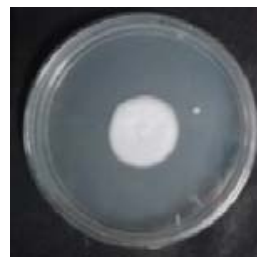

Cu(100ppm)

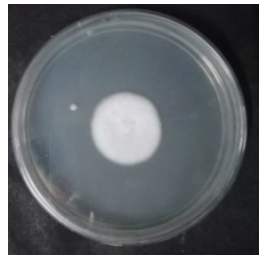

Mg(100ppm)

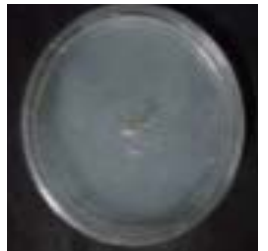

Zn(200ppm)

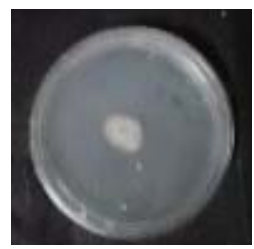

$\mathrm{Pb}(200 \mathrm{ppm})$

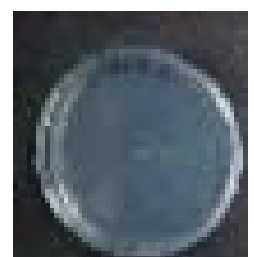

$\mathrm{Cu}(200 \mathrm{ppm})$

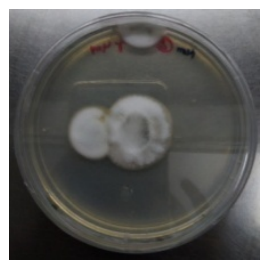

Mg(200ppm)

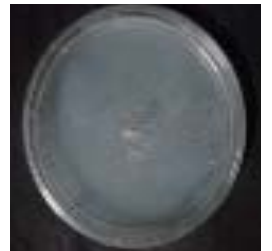

Zn(400ppm)

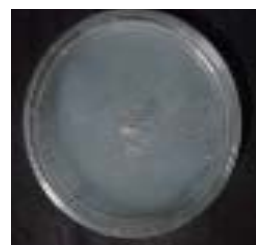

$\mathrm{Pb}(400 \mathrm{ppm})$

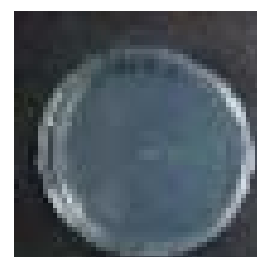

$\mathrm{Cu}(400 \mathrm{ppm})$

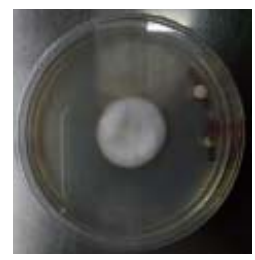

Mg(400ppm)

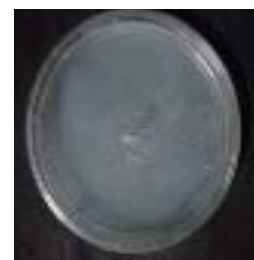

Zn(600ppm)

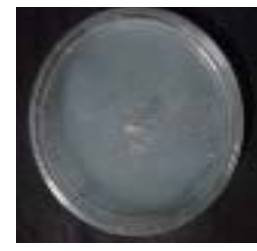

Pb(600ppm)

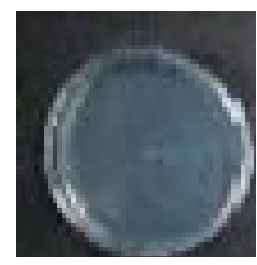

$\mathrm{Cu}(600 \mathrm{ppm})$

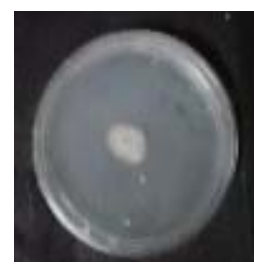

$\operatorname{Mg}(600 \mathrm{ppm})$

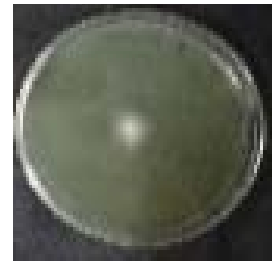

$\operatorname{Cr}(10 \mathrm{ppm})$

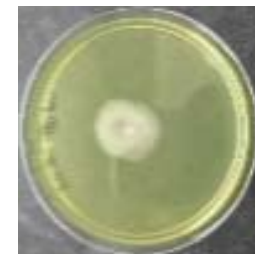

$\operatorname{Cr}(25 \mathrm{ppm})$

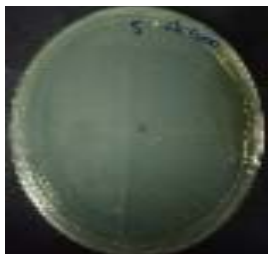

Cr(50ppm) 
Fig.4.7: Growth of S7 after exposure to different concentrations of heavy metal ions for 7-14 days.

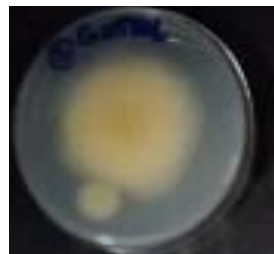

Control

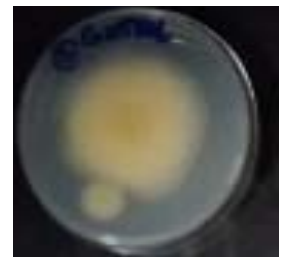

Control

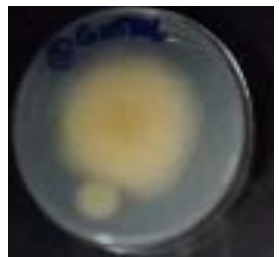

Control

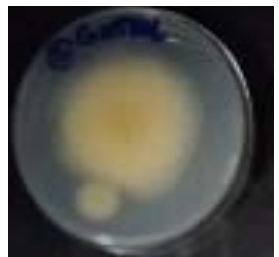

Control

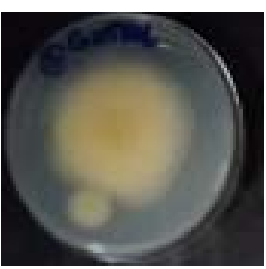

Control

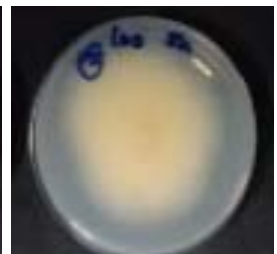

Zn(100ppm)

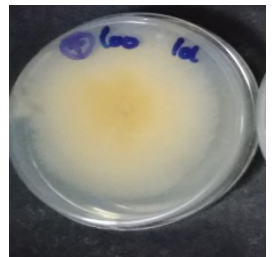

$\mathrm{Pb}(100 \mathrm{ppm})$

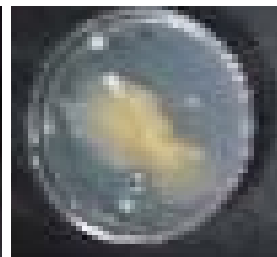

Cu(100ppm)

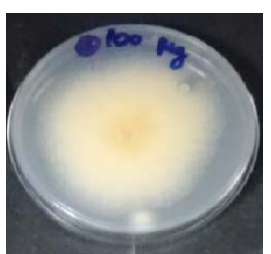

$\operatorname{Mg}(100 \mathrm{ppm})$

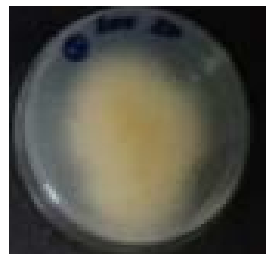

Zn(200ppm)

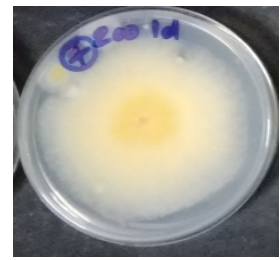

$\mathrm{Pb}(200 \mathrm{ppm})$

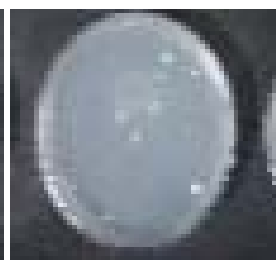

Cu(200ppm)

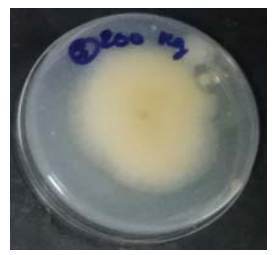

Mg(200ppm)

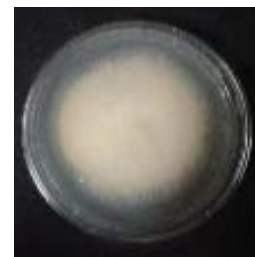

Zn(400ppm)

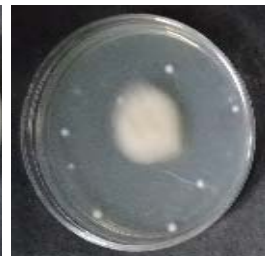

Pb(400ppm)

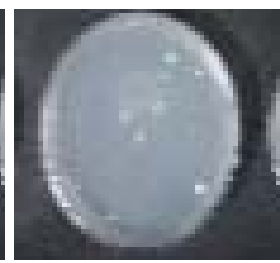

Cu(400ppm)

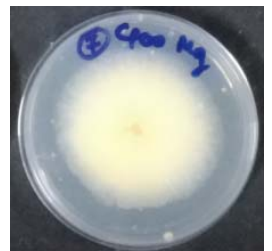

Mg(400ppm)

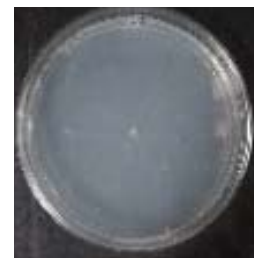

Zn(600ppm)

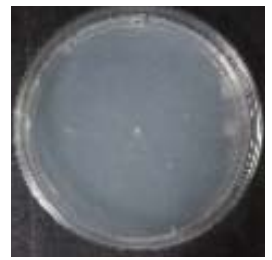

Pb(600ppm)

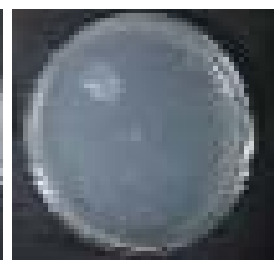

$\mathrm{Cu}(600 \mathrm{ppm})$

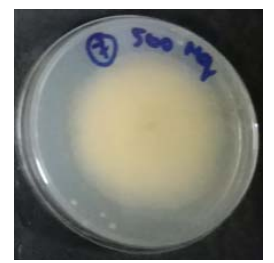

$\operatorname{Mg}(600 \mathrm{ppm})$

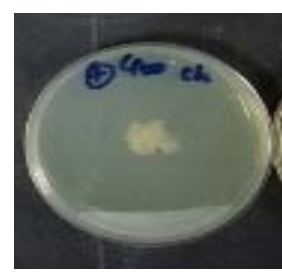

Cr(10ppm)

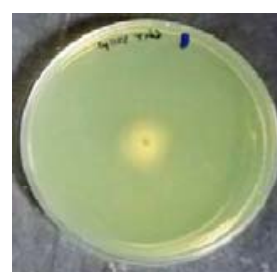

Cr(25ppm)

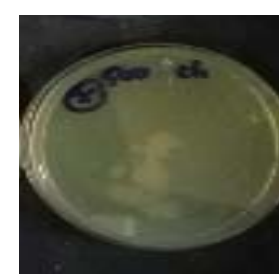

Cr(50ppm) 


\section{Discussion}

Contaminated sites are major sources of heavy metal resistant microorganisms. Due to prolonged exposure of plants to heavy metals there are modifications in their microbial populations. Heavy metal pollution of the soil leads to a decrease in microbial diversity. This is because some species that are sensitive to the imposed stress and due to the enhanced growth of other resistant species are unable to survive and become extinct gradually.

Due to the metal binding properties of their cell wall materials, Fungi are known to tolerate heavy metals readily [4]. Endophytes acquire tolerance and heavy metal resisting ability from the host as they live in close relation to the host. Hence the study was aimed to isolate heavy metal tolerant endophytic fungi from a plant grown in polluted sites.

The endophytic fungi were isolated from leaves of $T$. procumbens grown on industrial polluted site which is polluted with heavy metals and other pollutants in the soil for several years. Fungi isolated as per previous International taxonomic keys belonged to the genera Fusarium, Aspergillus sp. (Aspergillus flavus, A. niger), Curvularia, and unidentified isolates 6 \& 7. Further confirmation of the strains should be made by sequencing analysis. All the isolates were screened for tolerance against zinc, lead, copper, magnesium, and potassium dichromate starting at a concentration of $5 \mathrm{ppm}$ to $600 \mathrm{ppm}$. Aspergillus sp and Fusarium sp. could tolerate lead concentration of $600 \mathrm{ppm}$. Curvularia sp and Isolate 7 could tolerate to a lesser degree with MIC value of $400 \mathrm{ppm}$.

Cr showed the strongest inhibition towards all the isolates at a concentration of $50 \mathrm{ppm}$. The majority of the tested fungi were unable to grow in the presence of this metal and were sensitive to the presence of this metal ions in the growth. Zinc at a concentration of $600 \mathrm{ppm}$ was tolerated by Aspergillus sp and at 400 ppm by strain 7 and to a lesser degree by other isolates. Copper at a concentration of $400 \mathrm{ppm}$ was tolerated by Fusarium sp and to a lesser concentration of $200 \mathrm{ppm}$ by other isolates.

The screening test showed diversity in the heavy metal tolerance of the isolates. Few researchers have reported similar results [4], [11]. There were variations in resistance against individual metals among the isolates. The results indicate that the resistance level against individual metals was dependent on the isolates and different species of fungus showed different tolerance pattern. Some were sensitive, moderately tolerant, and tolerant and varied with different metals. Isolates of the same genus also showed a considerable difference in metal resistance level.

Noticeable differences in $\mathrm{Cu}, \mathrm{Zn}$, and $\mathrm{Cr}$ tolerance have also been found among the isolates. This differences in metal tolerance shown by different isolates may be due to difference in tolerance processes and resistance mechanisms. Few isolates of Aspergillus flavus were more tolerant for both metals ( $\mathrm{Cr} \& \mathrm{~Pb}$ ) while others were very sensitive to $\mathrm{Cr}$ but tolerant to $\mathrm{Pb}$.

\section{Conclusion}

As the heavy metals have toxic effects on human, animal, and plant health. It is essential to minimize heavy metal concentrations in the environment. Microbial remediation or 
bioremediation has the advantages of the removal of large amounts of heavy metal efficiently at a low cost. Endophytic fungi show excellent metal-binding capacity and have the ability to protect the host against heavy metal toxicity. Hence in this study endophytic fungi from the leaves of Tridax procumbens collected from five different industrial polluted sites were tested for their tolerance and ability to remove heavy metals from the solid medium in lab condition. The isolated fungus showed appreciable potential to grow in the presence of $\mathrm{Cr}, \mathrm{Zn}, \mathrm{Pb}, \mathrm{Cu}$, and $\mathrm{Mg}$ and some isolates could tolerate heavy metals up to $600 \mathrm{ppm}$. Further investigations are needed to confirm the isolates.

Acknowledgment: We thank Dr. I. Seetha Lakshmi, Lifeteck Research Centre, Chennai for providing lab facilities.

\section{Conflict of interest: None}

\section{References}

1. Saima Saif, and Mohammed Saghir Khan., 2017, “Assessment of Heavy Metals Toxicity on Plant Growth Promoting Rhizobacteria and Seedling Characteristics of Pseudomonas putida SFB3 Inoculated Greengram”, Acta Sci. Agric., 1(2), pp. 47-56.

2. Aishwarya Sani, Venkateswarulu Nagam, Vasudeva Reddy Netala, Vijaya Tartte., 2017, “Characterization of Heavy Metal Resistant Endophytic Fungi from Boswellia Ovalifoliolata”, Int. J. Sci.,3, pp. 1072-1076.

3. Naziha M Hassanein, Mervat MA El-Gendy, Hussein Abd El-Hay Ibrahim, Doaa H Abd El Baky.,2012, "Screening and Evaluation of Some Fungal Endophytes Of Plant Potentiality as Lowcost Adsorbents For Heavy Metals Uptake From Aqueous Solution”, Egypt J. exp. Biol., 8, pp. 1723.

4. Ezzouhri, L., Castro, E., Moya, M., Espinola, F. and Lairini K., 2009, "Heavy Metal Tolerance Of Filamentous Fungi Isolated From Polluted Sites in Tangier, Morocco”, Int J. Sci. Res., 3, pp. 035048.

5. Atish K Maldhure., 2015, “Tridax procumbens medicine for life”, Int J. Pharma. Bio. Sci. 6, pp. 810-815.

6. Govarthanan, M., Mythili, R., Selvankumar, Kamala-Kannan, S., Rajasekar, Young-Cheol Chang A., 2016, "Bioremediation of heavy metals using an endophytic bacterium Paenibacillus sp. RM isolated from the roots of Tridax procumbens", Biotech pp.1-7.

7. Samantha Beck, Heather Mathison, Toma Todorov, Esli-Armando Calderon-Juarez and Olga R Kopp., “A review of medicinal uses and pharmacological activities of Tridax procumbens $(L)$ ”, J. Plant Stud., 17, pp. 19-35.

8. Shahnawaz Ahmad Mir, Zubair Jan, Shafia Mir, Ayaz Mahmood Dar, and Gouri Chitale., 2017, “A Concise Review on Biological Activity of Tridax procumbens Linn.”, Organic Chem. Curr. Res., 6, pp. 1-4. 
9. Sani Aishwaryai, Venkateswarulunagam, Vasudeva Reddy Netala, Vijaya Tartte.,2017, "Screening and identification of heavy metal-tolerant endophytic fungi Lasiodiplodia theobromae from Boswellia ovalifoliolata an endemic plant of Tirumala hills”, Asian J. Pharm. Clin. Res., 10, pp. 488-491.

10. Xinya Li, Wei Li, Long Chu, James F White Jr, Zhi Xiong, and Haiyan Li., 2016, "Diversity and heavy metal tolerance of endophytic fungi from Dysphania ambrosioides, a hyperaccumulator from Pb-Zn contaminated soils”, J. Plant Interact. 1, pp.186-192.

11. Zafar, S., Aqil, F., Ahmad., I., 2007, "Metal tolerance and biosorption potential of filamentous fungi isolated from metal contaminated agriculture soil”, Bioresour. Technol., 98, pp. 2557-2561. 